\title{
Advances in Dry Eye Disease
}

\author{
An expert interview with Cynthia Matossian
}

Matossian Eye Associates, Pennington, NJ, USA

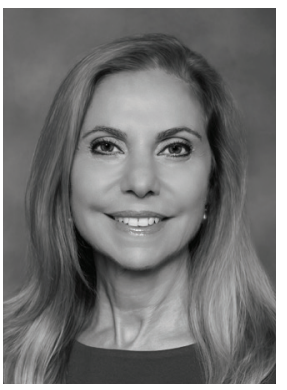

\section{Cynthia Matossian}

Cynthia Matossian, MD, FACS, is the Founder and Medical Director of Matossian Eye Associates, an integrated ophthalmology and optometry private practice with locations in New Jersey and Pennsylvania. She specializes in refractive cataract surgery and ocular surface disease. In November, she was presented with the Ophthalmic World Leaders (OWL) Visionary Award in recognition of how she has paved the way for advancement through diversity in her field. It is a great honor to be bestowed with this award as they are based on peer votes.

DOl: https://doi.org/10.17925/USOR.2019.12.1.13

\section{Keywords}

Dry eye disease, treatment adherence

Disclosure: Cynthia Matossian is a consultant to Allergan, Takeda/Shire, Imprimis, Quidel, J\&J for LipiFlow, and Ocular Therapeutix.

Review Process: This is an expert interview and as such, has not undergone the journal's standard peer review process.

Compliance with Ethics: This article is an opinion piece and does not report on new clinica data, or any studies with human or animal subjects performed by any of the authors.

Authorship: The named author meets the Internationa Committee of Medical Journal Editors (ICMJE) criteria for authorship of this manuscript, takes responsibility for the integrity of the work as a whole, and has given final approval for the version to be published.

Received: April 10, 2019

Published Online: April 23, 2019

Citation: US Ophthalmic Review. 2019;12(1):13-4

Corresponding Author: Cynthia Matossian,

Matossian Eye Associates, Pennington, NJ, USA

E: cmatossian@matossianeye.com

Twitter: @MatossianEye

Support: No funding was received in

the publication of this article.
Dry eye disease (DED) is one of the most common conditions encountered by ophthalmologists, with up to $50 \%$ of patients reporting symptoms. ${ }^{1}$ For many decades, DED was thought to be a simple condition caused by reduction of the aqueous phase of the tear film, but it has been redefined as: "a multifactorial disease of the tears and ocular surface that results in symptoms of discomfort, visual disturbance, and tear film instability with potential damage to the ocular surface, accompanied by increased osmolarity of the tear film and inflammation of the ocular surface." ${ }^{\prime 2}$ Treatment options for DED includes artificial tears, lipid-containing lubricants, liposomal spray, inserts, anti-inflammatory or immunosuppressant drops, antibiotics, oral dietary omega-3 essential fatty acids, amniotic membrane drops, autologous serum, intense-pulsed-light, thermal pulsation therapy, punctual plugs, moisture-retaining spectacles, hydrophilic bandage contact lenses, amniotic membrane lenses, and secretagogues. ${ }^{3}$ However, some of these treatment options have limited efficacy for the cell-mediated immune response and inflammation associated with DED. Only two drugs-cyclosporine and lifitegrast-are approved for the treatment of DED, and the disease represents an area of high unmet medical need, with a significant number of patients not receiving treatment.

In an expert interview, Cynthia Matossian discusses the challenges of treating DED, together with the highlights of her recent research and provides advice for ophthalmologists aiming to attain leadership roles.

\section{Q. Could you give us some background behind the cyclosporine $0.1 \%$ DED study presented at Hawaiian Eye?}

For over a decade, cyclosporine A (Restasis ${ }^{\circledR}$, Allergan, Dublin, Ireland) has been the only drug available for the treatment of DED. ${ }^{4}$ A new drug, lifitegrast (Xiidra ${ }^{\oplus}$, Shire [now Takeda], Lexington, MA, USA), which has a different mechanism of action, has been more recently introduced. ${ }^{5}$ In the USA, access to these drugs through insurance systems can be challenging since a pre-authorization is often required. This means that practitioners have to demonstrate that the patient has failed alternative treatments such as artificial tears. This process is extremely time-consuming and is an additional burden on physicians and their staff. An alternative option is a new formulation: cyclosporine with chondroitin sulphate (Klarity-C, Imprimis, San Diego, CA, USA), which is available by direct pay model, meaning the patient does not go through their insurance company. The medication is provided three bottles at a time in order to minimize shipment costs, and delivered to the patient at home. The bottles cost around $\$ 50$ each. Many patients prefer this option as it is usually less expensive than their insurance plan. 
I wanted to learn more about this product so, along with two other physicians, Dr William Trattler and Dr Jennifer Loh, both of whom practice in Florida, and myself in Pennsylvania and New Jersey, we did a pilot study of about 30 patients. ${ }^{6}$ We looked at symptom improvement, ocular surface disease index (OSDI) score and corneal staining in each eye pre- and post-treatment initiation in patients who used this product twice daily over a 90-day period.

\section{Q. Can you talk us through the results so far? What are the next steps in terms of follow-up?}

The eye scores improved as an aggregate. At the start of the study, $62 \%$ of patients had an OSDI score of severe. This improved to $12 \%$ after 90 days. There was also a statistically significant improvement in corneal staining in patients taking cyclosporine/chondroitin sulphate over 90 days. One limitation of the study was that we didn't assess how patients ranked the level of burning and the feeling of the drug going into their eye. Anecdotal reports from the study participants suggest that the product was more comfortable to use and created less sting and burn on instillation compared to traditional cyclosporine products.

On the basis of these findings we are performing an extension study involving around 75-100 patients, with the aim of producing more robust data. Since this is an extension study, we cannot add any new parameters; however, in the future, we may consider adding additional variables.

\section{Q. What are the main challenges you face when treating patients with DED?}

There are many challenges. One key challenge for physicians attempting to treat DED is that some patients are asymptomatic. When I tell them they have DED, they react with disbelief because they don't feel anything. This is an issue when they have chronic, progressive disease and need lifelong medication to prevent the disease becoming symptomatic. Performing early diagnostic tests that can objectively demonstrate abnormal values to patients is critically important. I use imaging to demonstrate abnormalities in meibomian glands, which gets the message across, as well as a high osmolarity number or a positive InflammaDry test (Quidel, San Diego, California, USA) where the patient can see the red stripe, which means they have high levels of inflammatory biomarkers on the ocular surface. Showing objective data to patients helps them to believe they have DED, even if it is asymptomatic, and to accept treatment.

Another challenge is the number of patients who discontinue their treatment over time (this could be weeks or months). It is very difficult to adhere to daily treatment for a chronic disease. cost is also an issue in keeping people on therapy.

\section{Q. How do you see the treatment landscape changing to increase treatment adherence in DED?}

Current treatment involves one drop administered twice a day. If we can minimize the effort involved in treatment, we might get greater compliance. Researchers are currently evaluating inserts that would go into the inferior or superior puncta and deliver drugs over a period of time; at present the duration is 30 days. ${ }^{7}$ Recently an intracanalicular insert for dexamethasone (Dextenza ${ }^{\circledR}$, Ocular Therapeutix, Bedford, Massachusetts, USA) was approved by the FDA for the treatment of ocular pain after ophthalmic surgery. ${ }^{8}$ Perhaps this product could be used off-label for ocular surface disease during exacerbation of DED. This would not be suitable for long-term use as it is a steroid, but could provide relief to patients during flare-ups. $\square$
1. Stapleton F, Alves M, Bunya VY, et al. TFOS DEWS II Epidemiology Report. Ocul Surf. 2017;15:334-65.

2. The definition and classification of dry eye disease: report of the Definition and Classification Subcommittee of the International Dry Eye WorkShop (2007). Ocul Surf. 2007:5:75-92.

3. Al-Saedi Z, Zimmerman A, Bachu RD, et al. Dry eye disease: present challenges in the management and future trends. Curr Pharm Des.
2016;22:4470-90.

4. Kymionis GD, Bouzoukis DI, Diakonis VF, et al. Treatment of chronic dry eye: focus on cyclosporine. Clin Ophthalmol. 2008;2:829-36

5. Donnenfeld ED, Perry HD, Nattis AS, et al. Lifitegrast for the treatment of dry eye disease in adults. Expert Opin Pharmacother. 2017;18:1517-24.

6. Study shows cyclosporine emulsion improves dry eye signs, symptoms. Presented by Cynthia Matossian at Hawaiian Eye/
Retina 2019, January 19-25, Waikoloa Village, HI, USA

7. Vickers LA, Gupta PK. The future of dry eye treatment: a glance into the therapeutic pipeline. Ophthalmol Ther. 2015;4:69-78.

8. Healio, Ocular Surgery News. Supplemental NDA accepted for Dextenza inflammation indication. Available at: www.healio.com/ ophthalmology/cataract-surgery/news/online/\%7B1f30729f-dc6b4a54-9bc4-3499c3177ed2\%7D/supplemental-nda-accepted-fordextenza-inflammation-indication (Accessed 5 April 2019). 